\title{
Photodegradation of selected $\pi$-conjugated electro-optic chromophores
}

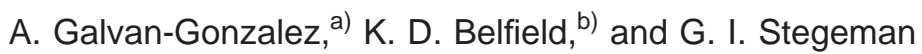

School of Optics and CREOL, University of Central Florida, 4000 Central Florida Boulevard, Orlando, Florida 32816

M. Canva ${ }^{\mathrm{c})}$

Laboratoire Charles Fabry de l'Institut d'Optique, UMR 8501 CNRS, Université d'Orsay / Paris-XI, 91403 Orsay Cedex, France

S. R. Marder, K. Staub, and G. Levina

Beckman Institute, California Institute of Technology, Pasadena, California 91125 and Department of Chemistry, University of Arizona, Tucson, Arizona 85721

R. J. Twieg

Department of Chemistry, Kent State University, Kent, Ohio 44242

(Received 12 December 2002; accepted 8 April 2003)

The photodegradation properties of six electro-optic chromophores in guest-host polymer formats were measured as a function of wavelength throughout the visible and up to $1.3 \mu \mathrm{m}$ in the near infrared. The chromophore structure was varied to provide insight into its relationship with photostability. (C) 2003 American Institute of Physics. [DOI: 10.1063/1.1578703]

\section{INTRODUCTION}

The systematic development of charge transfer chromophores for electro-optic applications has occurred over the last two decades. ${ }^{1}$ Typically, electro-optic molecules consist of an electron donor and an acceptor group separated by a conjugated "bridge" that facilitates charge transfer between the two terminating groups. This substitution pattern leads to an asymmetry in the charge distribution in the electronic ground state and, hence, to the large permanent ground state dipole moments that are required for molecular alignment via electric field poling. Furthermore, the large changes in the charge distribution in the first excited state of the molecule relative to the ground state result in a large secondorder optical nonlinearity, i.e., the electro-optic coefficient responsible for a change in the refractive index when an electric field is applied.

The chromophore DANS (4-N,N-dimethylamino4 ' nitrostilbene, $\mathrm{Me}_{2} \mathrm{~N}-\mathrm{Ph}-\mathrm{CH}=\mathrm{CH}-\mathrm{Ph}-\mathrm{NO}_{2}$, Fig. 1) has historically been considered as the prototype for the stilbene family of electro-optic chromophores. Early work at Celanese (now Hoechst-Celanese) showed that intentional photobleaching of DANS in the visible-UV (the primary absorption bands) could be used to fabricate channel waveguides. When attached as a sidechain group to a methacrylate backbone polymer DANS was one of the first chromophores investigated and implemented in an electro-optic polymer waveguide device. ${ }^{2-5}$ Here, the channel region was masked off during illumination in the visible and the refractive index of the surrounding material reduced by photochemical reactions, leaving the channel with a large refrac-

\footnotetext{
${ }^{a}$ Present address: Agere Systems, 4656 Towerwood Drive, Brownsville, TX 78521.

b) Also with: Department of Chemistry.

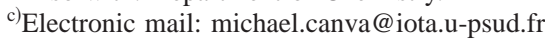

tive index and, hence, forming a waveguide. Electro-optic modulators based on this material and process were demonstrated. ${ }^{4}$

This same group later demonstrated that photobleaching also leads to photodegradation of DANS even in the 1300 $\mathrm{nm}$ communications band (well away from the primary electronic transitions near $500 \mathrm{~nm}) .{ }^{6}$ When DANS waveguides were used around $1300 \mathrm{~nm}$, a guided wave transmission loss resulted from photobleaching of the channel by the guided light. The largest effect occurred at $1280 \mathrm{~nm}$, the wavelength associated with the singlet oxygen absorption peak. At 1300 $\mathrm{nm}$ the degradation was a factor of two slower. ${ }^{7,8}$ However, in the compound 4-N,N-dimethylamino-4' -nitrobiphenyl $\left(\mathrm{Me}_{2} \mathrm{~N}-\mathrm{Ph}-\mathrm{Ph}-\mathrm{NO}_{2}\right.$, Fig. 1) where the $-\mathrm{C}=\mathrm{C}-$ unit connecting the two phenyl groups in DANS is now replaced by a single bond, the photodegradation was dramatically reduced. Subsequent work in an atmosphere purged with nitrogen showed an order of magnitude increase in the photostability of DANS at $1320 \mathrm{~nm}$, further implicating the participation of oxygen in the photodegradation (believed to be primarily due to the reaction at and scission of the carbon double bond in the central bridge). ${ }^{9}$ It was later found by other researchers that the photodegradation rate can be accelerated by the addition of a photosensitizer. ${ }^{10}$ DANS waveguide transmission losses were compared at $1320 \mathrm{~nm}$ and at $1550 \mathrm{~nm}$ and no degradation at $1550 \mathrm{~nm}$ was found on the limited time scale of the experiment. ${ }^{8}$ However, it must be noted that the projected lifetime of DANS at $1320 \mathrm{~nm}$ is still only a few days at an average illumination of $1 \mathrm{~mW}$ over $10 \mu \mathrm{m}^{2}$ and at least three orders of magnitude improvement is necessary to give a lifetime of ten years at $1550 \mathrm{~nm} .^{11,12}$

More details about the photodegradation characteristics of DANS including the possible effects of two-photon absorption were reported in a series of letters in the late 1990s. ${ }^{11-13}$ The concentration of molecules (related to the magnitude of the strongest charge-transfer state) versus 


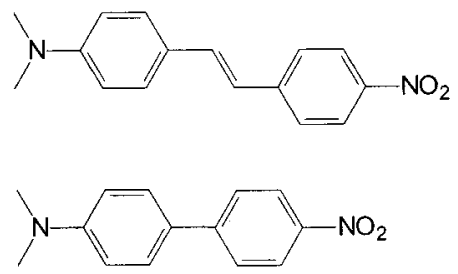

FIG. 1. Structure of typical nonlinear optical electro-optic chromophores. The stilbene chromophore DANS (top) and the analogous biphenyl derivative (bottom) have different conjugated bridges but identical donor and acceptor groups.

illumination time was monitored by measuring changes in the absorption spectrum. Parameters including the difference between sidechain attachment and guest-host inclusion, the wavelength dependence of the photodegradation from 400 to $1300 \mathrm{~nm}$ and the temperature and atmosphere dependence were all examined. ${ }^{12}$ The photodegradation figure-of-merit was found to increase by seven orders of magnitude with increasing wavelength (a minimum at the absorption maximum of the strong charge-transfer state near $470 \mathrm{~nm}$ and then a steady increase until a plateau and then a decrease is encountered at $1320 \mathrm{~nm}$ due to singlet oxygen).

There are two clear and dominant photodegradation pathways for stilbenes: trans-cis isomerization and the creation of chemical species that involve oxygen induced chemical reactions primarily at the central stilbene $\mathrm{C}=\mathrm{C}$ bond. ${ }^{12}$ Some of the reactions that may occur are illustrated in Fig. 2. The first process does not require oxygen. Here, trans-stilbene is first isomerized upon irradiation to the thermodynamically less stable cis-stilbene isomer. A subsequent photoinduced isomerization of the cis-stilbene isomer via a conrotatory, six-electron electrocyclic ring closure affords the trans isomer of a dihydrophenanthrene derivative. This tricyclic polyene is not aromatic and is unstable and subject to oxidation to the phenanthrene derivative and other additional photochemistry (not shown). There is no true trans-cis stilbene photostationary state established because the cis isomer population is gradually drained by the photocyclization process leading to phenanthrene. The second reaction class requires the presence of oxygen. Here, irradiation of the stilbene (or other polyene or condensed aromatic such as phenanthrene) can sensitize the formation of reactive singlet oxygen $\left({ }^{1} \mathrm{O}_{2}\right)$ by triplet energy transfer from an excited triplet state. The singlet oxygen thus formed can then add to the $\mathrm{C}=\mathrm{C}$ double bonds (as found in stilbene), resulting in formation of an oxetane that can undergo subsequent irreversible thermal reactions (not shown). These different and complex pathways can only be unraveled by chemical analysis of the photoproducts. This might be accomplished by a combination of chromatographic methods (high pressure liquid chromatograpy or gas chromatography coupled with an analytical means such as mass spectrometry. Using these techniques is should be possible to determine the evolution of degradation in the samples, i.e., determining both primary and secondary photoproducts. Unfortunately the requisite hardware was not available to us and the analysis was beyond the scope of this work. Furthermore, two charge-transfer states contribute to the measured photodegradation.

These results for DANS have raised questions about the photostability of other alkene containing chromophores and

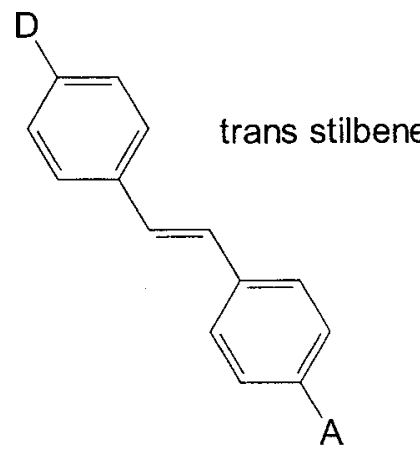

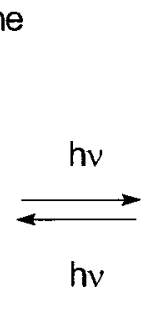

cis-stilbene

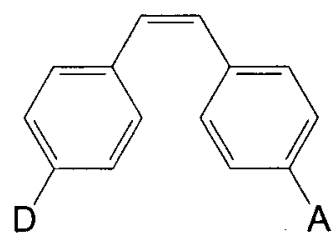

hv
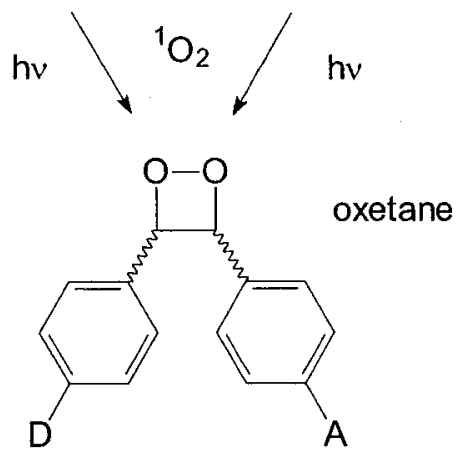

dihydrophenanthrene

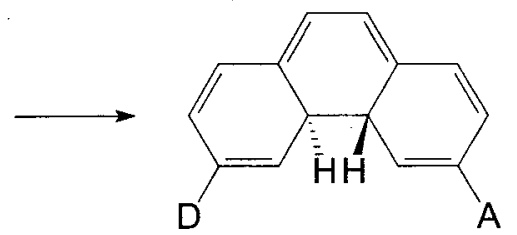

[O]

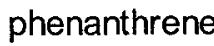

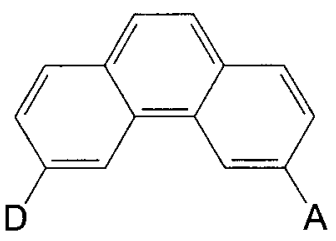

FIG. 2. Photochemical reactions of stilbene derivatives. The $\mathbf{D}$ and $\mathbf{A}$ are substituents, e.g., electron-donor $D$ and electron-acceptor $A$ groups. 


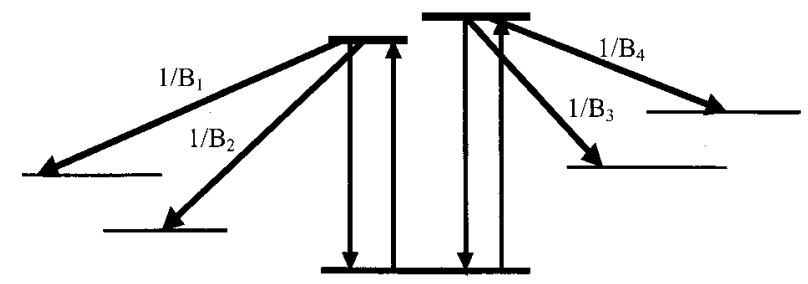

FIG. 3. A schematic of the photodegradation model found for DANS. There are two charge transfer states that participate, and there are at least two photodegradation pathways from each.

polymers. ${ }^{14}$ In contrast to other studies of polymer figuresof-merit for electro-optics, changes in the figures-of-merit for photostability with changes in chromophore structure are currently difficult to interpret, limiting the predictive power of simple structure-property relationships. For example, it has proven possible to evaluate the strength of different electron donor and acceptor groups and the electronic transmission capabilities of different $\pi$ groups so as to design molecules with predictably large nonlinearities. However, in the case of photostability, there is much less rigorous information relating structure to photodegradation that can be triggered at many different sites in a given chromophore, depending on the local polymer environment, the range of chemical reactions that can occur, and the presence of oxygen, etc. In order to assess whether the $\mathrm{C}=\mathrm{C}$ functionality as found in stilbene is the "Achilles heel" of $\pi$-conjugated chromophores, we investigated the photostability of a number of selected alkene containing chromophores. Other work conducted on the chromophore "DCM" previously addressed the issue of the stability of such a bond in this laser dye. ${ }^{15,16}$

\section{PHOTODEGRADATION MODEL USED AND PARAMETERS MEASURED FOR DANS}

Assuming that the results for DANS are prototypical of other alkene containing chromophores, the states and pathways expected to participate in the photodegradation of $\pi$-conjugated chromophores are illustrated in Fig. 3. This process typically starts with the absorption of a photon and the excitation of a fraction of the chromophores into one of the two lowest lying excited charge-transfer states of the molecule. ${ }^{17}$ Those chromophores that return to the original ground states on de-excitation do not contribute to the photodegradation. However, any other de-excitation pathway leading to a different final state (and usually different chemical species) results typically in a loss of overall conjugation with a corresponding reduction of the electro-optic nonlinearity of the molecule and a reduction of refractive index of the medium. For example, reversible trans-cis isomerization in the excited state changes the geometric structure of the molecules, or an irreversible chemical reaction such as photo-oxidation leads to one or more new chemical species. Many other processes can occur for any given chromophore and the total photodegradation phenomenon can be very complex, involving multiple intermediate steps before the final photodegradation products are reached. ${ }^{18,19}$ The new altered chemical or geometric structure likely involves some reduction of the effective conjugation in the molecule or changes in the characteristics of the donor or acceptor groups responsible for the optical nonlinearity. These changes represent serious problems for most photonic applications. ${ }^{19-23}$

The figure-of-merit which describes a single photodegradation process from a single charge-transfer excited state is given by $B / \sigma$ where $B^{-1}$ is the probability that photodegradation occurs from the excited state, and $\sigma$ is the molecular absorption coefficient. Larger values of $B / \sigma$, lead to slower photodegradation rates. In the case of DANS with multiple pathways and multiple excited states, (as in the case of multiple decay mechanisms with different time constants where there is an effective decay time constant which inverse is the sum of all inverse), there is an effective $B, B_{\text {eff }}$, with $B_{\text {eff }}^{-1}$ $=\sigma_{1} / \sigma_{T} B_{1}+\sigma_{2} / \sigma_{T} B_{2}+\cdots$ where $\sigma_{i}$ is the absorption coefficient associated with the $i$ th excited state (which depends on photon energy differently for each state), $\sigma_{T}=\sigma_{1}$ $+\sigma_{2} \cdots$, and the $B_{i}^{-1}$ contain the combined quantum efficiencies for all degradation pathways from the $i$ th state (that is because $B / \sigma$ is the quantum analog of the time constant).

In any photodegradation experiment, the concentration of molecular species starting from ground states is monitored by either measuring the reduction in the full absorption spectrum, or by monitoring the change in absorption at a specific wavelength, usually in the tail of an absorption line of the molecule under study and in a transparent region of the photoproduct(s). ${ }^{17,24}$ The initial slope of the change in the sample transmission with integrated optical fluence is directly proportional to $\sigma_{T} / B_{\text {eff }}$ (i.e., the rate of change is proportional to the number of excited molecules, via $\sigma_{T}$, which effectively changes conformation, with a probability $1 / B_{\text {eff }}$ and therefore provides a direct measurement of this figureof-merit. The maximum complexity that can be unraveled by the experiments described here is the existence of two participating charge transfer states, and the identification of two pathways providing one is photo-oxidation. Identifying photo-oxidation as a pathway requires measuring and comparing the effects of photodegradation in air (or oxygen) and in a nitrogen atmosphere. The variation in $B$ with wavelength is the signature of multiple degradation pathways. In fact, this has been found for DANS, ${ }^{12}$ and we thereafter summarize the data that had been collected, and for most of it published in greater details, as it is a typical example of what can happen,

From the wavelength dependence of $B$ in DANS it was clear that there are two values of $B$, with the quantum efficiency $B_{2}\left(3 \times 10^{4}\right)$ for photodegradation associated with a higher energy charge-transfer state, and the smaller quantum efficiency $B_{1}\left(8 \times 10^{5}\right)$ with the lowest energy chargetransfer state. This is manifested by a clear transition in the 500-600 $\mathrm{nm}$ wavelength range between two wavelengthindependent plateaus for $B .^{12}$

Observation of the visible-near UV DANS absorption spectrum during illumination showed that there are two spectral components at 2.9 and $4.1 \mathrm{eV}$ that are photobleached efficiently by $544 \mathrm{~nm}$ radiation in air. ${ }^{12}$ The first peak corresponds to the electronic transition from the ground state to the lowest energy singlet excited state $\left(S_{1}\right)$ with strong charge transfer characteristics. The second corresponds to 
transitions from the ground state to another excited chargetransfer $\left(S_{2}\right)$ that involves large bond-order modifications and, hence, more pronounced geometric deformations. ${ }^{25}$ Theoretical calculations have shown that these two absorption spectra are associated with two separate charge-transfer states. A photoproduct appears with a peak at $380 \mathrm{~nm}$. Similar photobleaching curves are obtained in an oxygen atmosphere. However, in a nitrogen atmosphere, the behavior is quite different for short times with primarily the dominant charge-transfer peak undergoing photodegradation and transferring its oscillator strength into a new spectral feature at $360 \mathrm{~nm}$, presumably due to the cis isomer and a weaker feature at $240 \mathrm{~nm}$. At later times, the $360 \mathrm{~nm}$ feature is photobleached and the broad absorption centered at $240 \mathrm{~nm}$ continues to grow. When oxygen then replaces the nitrogen, the final photoproducts are different from those obtained when air or oxygen was used from the outset. Furthermore, in separate experiments, the photoproducts in air are different for bleaching at 488 vs $544 \mathrm{~nm}$ (and at 458 vs $515 \mathrm{~nm}$ ), showing the complexity of the photodegradation processes involved. $^{18}$

As detailed in previous communications, ${ }^{24}$ the measured or extrapolated knowledge of the $B / \sigma$ parameter at a given wavelength allows evaluating the lifetime limitation of a device due to such cumulative photodegradation linear effect, knowing the device geometric structure and illumination conditions. In all case, for a given device such lifetime limitation will scale with $B / \sigma$. As example, the best sample we studied so far was a modified disperse-red one doped polymer, and we extrapolated that a $10 \mu \mathrm{m}^{2}$ section waveguide would have a lifetime of about a year if continuously operated at $1064 \mathrm{~nm}$ with an average power of $1 \mathrm{~mW}^{26}$

\section{EXPERIMENTAL RESULTS FOR $\pi$-CONJUGATED CHROMOPHORES}

The chemical structures of the chromophores 1-6 studied here are shown in Fig. 4. Chromophores 1-5 all contain an olefinic bridge while chromophore 6 contains an acetylene bridge. Chromophores 1 and 2 are simple $D-A$ substituted stilbenes, chromophore 5 is a stilbene with an additional cyano acceptor on the olefinic bridge while 3 and 4 are trienes terminated with a donor amine substituted benzene ring and a pair of cyano acceptor groups. Table I contains a list of chromophores 1-6 studied in guest-host format along with their salient properties. The typical formulation was 5\% by weight of 1-6 as guest in poly(methylmethacrylate) (PMMA) as host. In the case of 2, poly(N-vinylcarbazole) (PVK) was also used as a host polymer. ${ }^{27}$

The shapes of the absorption spectra are all similar for the chromophores studied and representative examples are given in Fig. 5. There are three well-defined peaks. Based on DANS, the two lowest energy (longest wavelength) peaks correspond to charge-transfer excited states with the weaker one usually located $40-100 \mathrm{~nm}$ below the stronger one and whose location depends on the specific donor and acceptor groups. The phosphonate 2 exhibits a short wavelength $\lambda_{\max }(380 \mathrm{~nm})$ for the lower energy peak whereas 3 and 4 have longer wavelength absorption maxima between 500 and
1

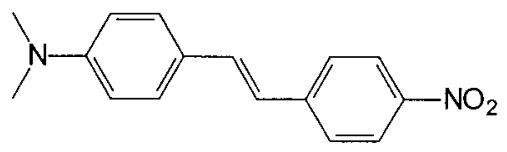

2

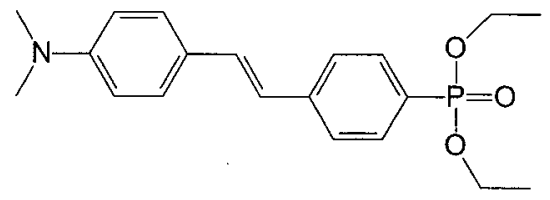

3

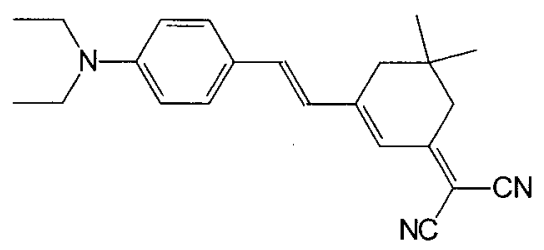

4

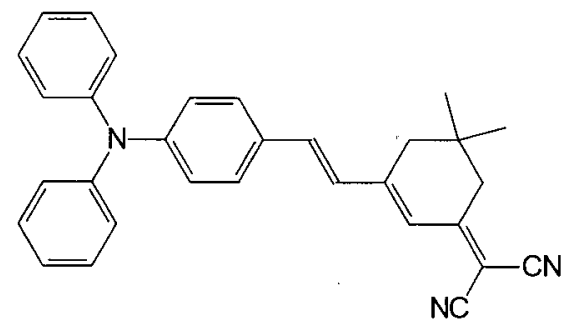

5

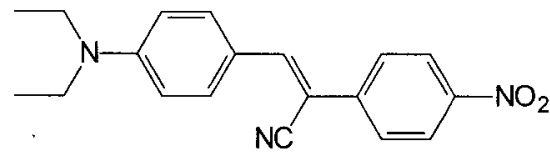

6

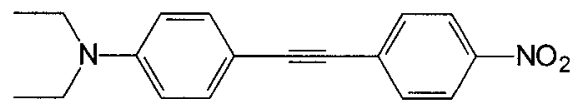

FIG. 4. Structures of the chromophores investigated in this study.

$530 \mathrm{~nm}$. This indicates a much stronger dominant charge transfer state in the latter trienes.

The evolution of the absorption spectrum during illumination in air and nitrogen at the long wavelength edge (442 $\mathrm{nm}$ ) of the dominant charge transfer was investigated for chromophore 2 in PMMA (Fig. 6). Because of the decreased acceptor strength associated with this phosphonate chromophore compared to DANS, all of the charge-transfer related peaks are moved to shorter wavelengths relative to DANS. (Here the higher energy charge-transfer state is believed to appear as the shoulder observed on the dominant absorption peak.) The decay of the main charge-transfer peak is slower in nitrogen than in air indicating that, just like in DANS, photo-oxidation is a contributing photodegradation mechanism. Furthermore, the changes in the spectrum at wavelengths about $100 \mathrm{~nm}$ below the dominant charge transfer absorption peak are also typical of what was observed previously in DANS. ${ }^{12}$ Namely, the peak at $270 \mathrm{~nm}$ grows monotonically in nitrogen. In both cases, absorption due to 
TABLE I. Chromophores studied and their properties including the wavelength for the peak of the absorption curve $\lambda_{\max }$ (determined on a thin film), the full width half maximum of the absorption curve, the $B$ parameters at 543 and $633 \mathrm{~nm}$, and the $D_{0}$ and $E_{0}$ fitting parameters. All of the chromophores $1-6$ were studied in PMMA and chromophore 2 was also studied in PVK.

\begin{tabular}{|c|c|c|c|c|c|c|}
\hline Chromophore & $\lambda_{\max }$ in $\mathrm{nm}\left[E_{\max }(\mathrm{eV})\right]$ & Full width at half maximum (nm) & $B @ 543 \mathrm{~nm}\left(\right.$ in $\left.10^{5}\right)$ & $B @ 633$ nm (in $\left.10^{5}\right)$ & $D_{0}\left(\right.$ in $\left.10^{36}\right)$ & $E_{0}(\mathrm{meV})$ \\
\hline 1 PMMA & $430(2.86)$ & 114 & 0.1 & 1 & 3 & 86 \\
\hline 2 PMMA & $370(3.36)$ & 60 & 0.6 & 0.8 & 5 & 130 \\
\hline 2 PVK & $390(3.19)$ & 100 & 1 & 1 & 70 & 91 \\
\hline 3 PMMA & $530(2.35)$ & 120 & 0.7 & 0.9 & 0.15 & 93 \\
\hline 4 PMMA & $500(2.48)$ & 130 & 1 & 3 & 1.3 & 93 \\
\hline 5 PMMA & $470(2.64)$ & 130 & 2 & 5 & 12 & 92 \\
\hline 6 PMMA & $430(2.86)$ & 110 & 1 & 6 & 1 & 117 \\
\hline
\end{tabular}

new photoproducts appears around $240 \mathrm{~nm}$. In summary, in this case, like DANS, it appears that more than one photodegradation mechanism is operative. As discussed in detail previously, ${ }^{24}$ based on the assumption that far from the main absorption band the $B$ parameter would only be due to one charge-transfer state and therefore independent of exciting photon energy, the the dependence of the figure of merit $B / \sigma$ in the near infrared would then only be due to the Voigt profiled red side line shape of the absorption band and could therefore be expressed in terms of the photon energy $E_{\text {phot }}$ as

$$
\begin{aligned}
B / \sigma & =B / \sigma_{0} \exp \left[-\left(E_{\mathrm{phot}}-E_{\mathrm{max}}\right) / E_{0}\right] \\
& =D_{0} \exp \left(-E_{\mathrm{phot}} / E_{0}\right), \\
D_{0} & =B / \sigma_{0} \exp \left[E_{\max } / E_{0}\right] .
\end{aligned}
$$

(It is for this reason that we plot the $B / \sigma$ data in terms of photon energy rather that wavelength.) This behavior effectively mirrors the dependence of the "red tail" of the absorption spectrum on the photon energy in this region of the spectrum. ${ }^{24}$ Here $E_{0}$ is a constant that varies with both the host polymer and the chromophore and the smaller the $E_{0}$, the spectrally narrower is the tail of the absorption spectrum and the faster the figure-of-merit increases with decreasing photon energy (i.e., increasing wavelength). Also $\sigma_{0}$ is the

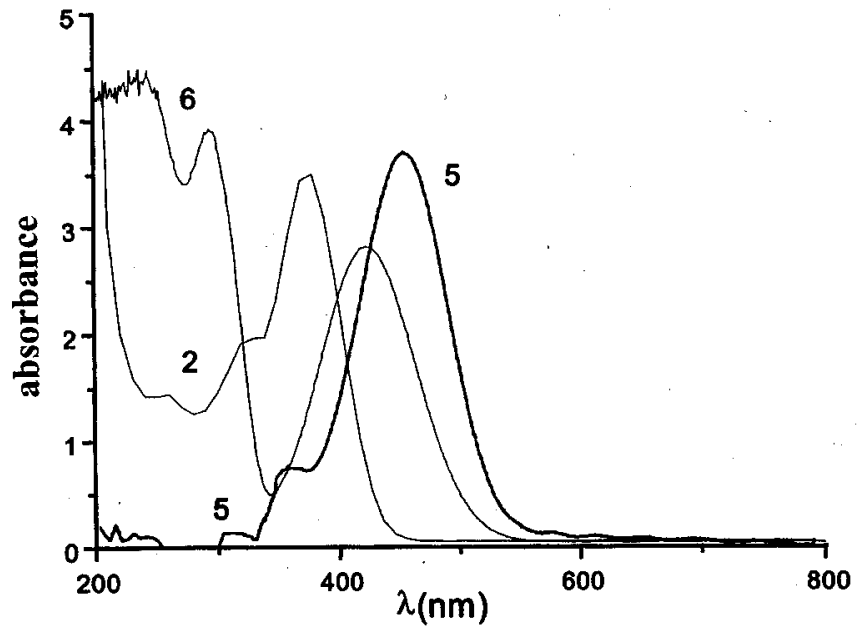

FIG. 5. Absorption spectra for three typical stilbene guest-host polymers, 2 (in PVK), 5 (in PMMA), and 6 (in PMMA). peak molecular absorptivity associated with the chargetransfer state located at $E_{\max }$. Here $D_{0}$ is proportional to the minimum lifetime per unit of photon flux. This relation has been proven to be valid for many electro-optic chromophores and is useful for predicting values of $B / \sigma$ in this spectral region with the knowledge of only two parameters $\left[E_{0}\right.$ slope and $D_{0}$ offset on $\log (B / \sigma)$ vs $h \nu$ representation]. ${ }^{18}$

The dependence of $B / \sigma$ on the photon energy $E_{\text {phot }}$ for all six chromophores (including DANS) is shown in Fig. 7. The associated error bars are not provided for these data.
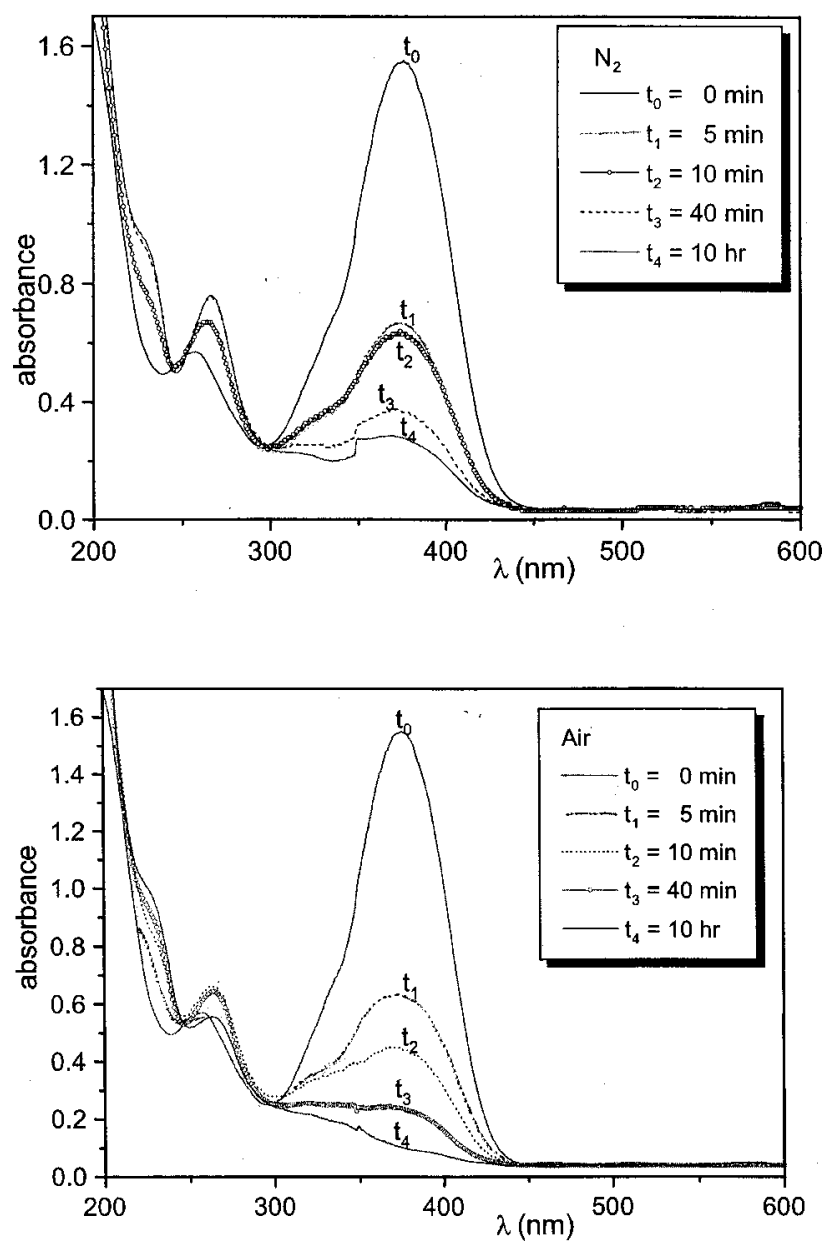

FIG. 6. The evolution of the absorption spectrum of $0.5-\mu$ m-thick film of 5 wt $\%$ of stilbene phosphonate 2 in a PMMA host polymer upon irradiation in nitrogen and air at $442 \mathrm{~nm}$. Here $t_{0} \ldots t_{4}$ correspond to irradiation times of 0 , $30,60,90$, and $120 \mathrm{~min}$, respectively. 


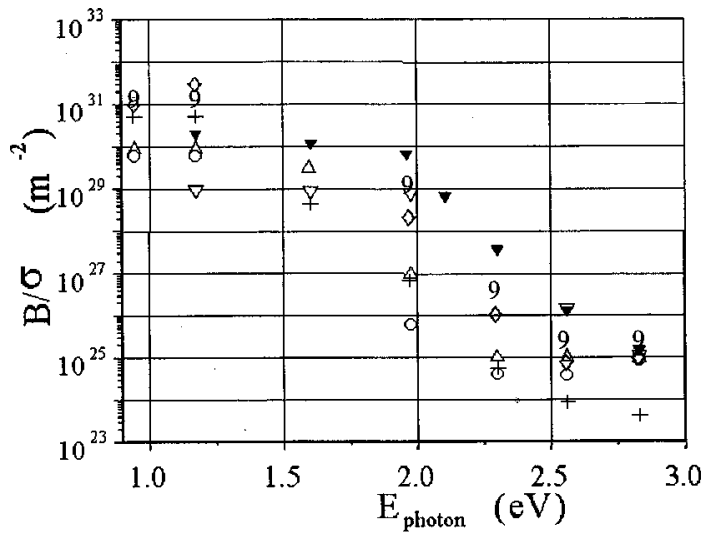

FIG. 7. The wavelength dispersion of the photodegradation figure of merit $B / \sigma$ for the chromophores (in PMMA unless otherwise indicated) $1(+)$, $2(\nabla), 2(\boldsymbol{\nabla}$, in PVK), $3(\bigcirc), 4(\triangle), 5(\diamond)$, and $6(9)$.

They are estimated to be fairly large, but even a factor of 2 would not change the conclusions as we are interested in variations over orders of magnitude as the photobleaching wavelength is varied over the visible and near-infrared spectrum. In general terms the shape of such variations are very similar, as noted previously for many other electro-optic chromophores. Furthermore, with the exception of the phosphonate 2, the dependence of $B / \sigma$ on $E_{\text {phot }}$ is quasilinear in the near infrared. However, there is a very clear leveling off of the $B / \sigma$ for energies below about $2 \mathrm{eV}(620 \mathrm{~nm})$ for the chromophore 2 with the phosphonate acceptor group studied in two different matrices. The unique aspect of the linear absorption spectra of phosphonate substituted stilbene 2 is that they are very narrow with a large $E_{\max }\left(\operatorname{small} \lambda_{\max }\right)$. As a result the absorption $\sigma$ decreases very rapidly with decreasing photon energy into the near infrared. Assuming a constant $B$ in this region of the spectrum, this leveling off of the figure-of-merit would be due to a leveling off of the absorption spectrum below $2 \mathrm{eV}$, as expected from a Voigt profile. (The Voigt profile has been found to give a good approximation to the absorption spectrum of DANS, the prototype stilbene. ${ }^{28}$ )

Chromophores, 3-5, have stronger charge-transfer states as evidenced by the shifts in the $E_{\max }$ to smaller photon energies (i.e., longer wavelengths). Of these, chromophore 5 is the most stable with $B / \sigma$ comparable to values reported previously for azobenzenes. This additional stability may be due to the fact that 5 has only a single trisubstituted alkene while 3 and 4 (a different class of chromophores) are trienes with more reactive multiple alkene functionality.

The physical parameter that describes the "strength" of the degradation pathways is $B$. It can be obtained from the measured $B / \sigma$ by measuring the molecular absorptivity $\sigma$ of the films using a Cary spectrophotometer. These measurements were used to generate the values of $B$ listed in Table I. They range from $B=10^{4}-2 \times 10^{5}$ at $543 \mathrm{~nm}$, and from 8 $\times 10^{4}$ to $6 \times 10^{5}$ at $633 \mathrm{~nm}$. This increase in $B$ in this spectral region was also observed in DANS and is a consequence of two excited states contributing to the photobleaching. The value at $633 \mathrm{~nm}$ is indicative of the value associated with the photostability of the lowest energy excited state. The value

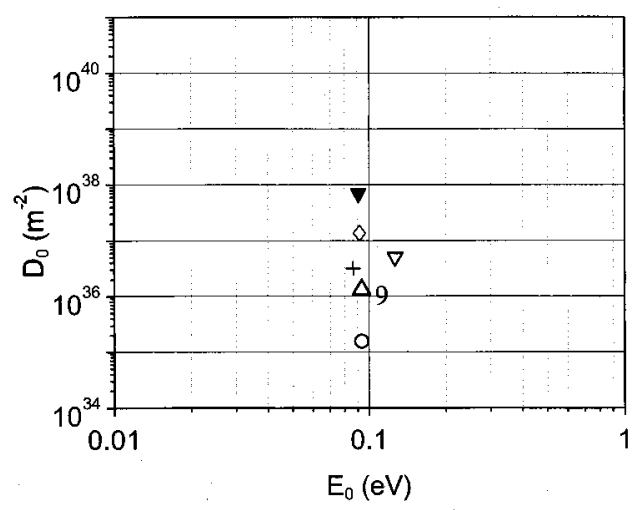

FIG. 8. Plot of $E_{0}$ vs $D_{0}$ for the chromophores, (in PMMA unless otherwise indicated) including $1(+), 2(\nabla), 2(\nabla$, in PVK), $3(\bigcirc), 4(\triangle), 5(\diamond)$, and 6 (9).

of $B$ in 5 is $5 \times 10^{5}$, significantly larger than that of DANS $\left(10^{5}\right)$, which does not have the cyano group.

The spread in $B$ in these chromophores is one order of magnitude, comparable to that found in azobenzenes. ${ }^{24}$ However, the median value of $B$ is typically a factor of 50 smaller than in the azobenzenes. However, it is important to note that it is a combination of $B$ and $\sigma$ that determines the figure-ofmerit. Since the absorption spectra are relatively narrow for some of the compounds, the figure-of-merit for the best compounds at $E_{\text {photon }}=1.4 \mathrm{eV}(1064 \mathrm{~nm})$ is comparable to that of azobenzenes.

In addition, the replacement of the diethylamino electron donor group in 3 by a diphenylamino group in 4 (everything else being unchanged) increases the photostability, $B=9$ $\times 10^{4}$ vs $3 \times 10^{5}$. This influence was also found in earlier in azobenzenes. ${ }^{24}$ The enhanced photostability of diarylamine versus dialkylamine donor chromophores may come from two contributions. First, often the diarylamine donor has a higher oxidation potential than the comparable dialkylamino donor compound. Second, in the diarylamino compound the hydrogens on carbon adjacent to the amine are absent. This site is the most reactive in the alkyl chain on the donor.

The range of the $E_{0}$ and $D_{0}$ for this family is shown in Fig. 8. As often the range of $E_{0}$ value (slope) is narrow and the values of $D_{0}$ (offset) spreads over several orders of magnitude. However, this is a classical example of the care that must be used in interpreting $E_{0}-D_{0}$ plots for predicting photostability. Here chromophore 2 in PVK has the largest $D_{0}$ and smallest $E_{0}$, normally a useful predictor for good photostability. But as is evident in Fig. 7, the low photon energy (longer wavelength) validity for these parameters is limited to about $2 \mathrm{eV}(633 \mathrm{~nm})$. So in fact chromophore 5, with the next highest $D_{0}$ and smallest $E_{0}$ is the most promising in this class of molecules.

The 4-N,N-diethylamino-4'-nitrotolane, chromophore 6, represents a substantial change from DANS in that the central carbon-carbon double bond, is replaced a carboncarbon triple bond. This has a large impact on the absorption spectrum, viz. Fig. 5. The higher energy absorption peak is actually comparable to or even larger than the lower photon energy peak that is the dominant one in DANS. This will 
certainly affect the decay processes, and this merits furtherinvestigation. Although the value of $B$ is larger than any found for the other chromophores, namely $B=6 \times 10^{5}$, the dependence of $B / \sigma$ versus wavelength is typical of the stilbenes, see Fig. 7. The acetylene group is prone to many of the reactions of stilbenes. The oxidation potential of an acetylene containing chromophore is generally higher than that of its stilbene counterpart.

\section{SECOND-HARMONIC GENERATION MEASUREMENTS OF PHOTOBLEACHED DANS SAMPLES}

The Maker fringe technique is a standard method for measuring the second order nonlinearity in thin films ${ }^{29}$ and it was used here to evaluate the loss of nonlinearity of DANS as a function of bleaching time. Thin film DANS samples were prepared by spin coating on substrates covered with a transparent indium tin oxide electrode followed by the vacuum deposition of an aluminum film onto the DANS surface. By applying electric field poling in a parallel plate geometry, the DANS molecules which have a large permanent dipole moment were partially aligned along the field direction. A nominal electric field of $100 \mathrm{~V} / \mu \mathrm{m}$ was used and no efforts were made to optimize the poling process. Finally, the electrode on the film's upper surface was etched away to allow optical beam access to the volume of the sample.

A $1064 \mathrm{~nm} \mathrm{Nd:} \mathrm{Yttritium-aluminum-garnet} \mathrm{laser} \mathrm{was}$ used in conjunction with a hydrogen Raman cell to produce $1579 \mathrm{~nm}$ radiation. We assume that the photodegradation at this wavelength can be neglected and the harmonic generated is too weak to lead to additional photodegradation. The 1579 $\mathrm{nm}$ optical beam was incident at variable angles from the surface normal and the second harmonic obtained on transmission was measured. The most reliable results were obtained for the $\operatorname{TM}(\omega) \rightarrow \operatorname{TM}(2 \omega)$ polarization combination. The standard Maker fringe analysis was performed on the data and the variation of the nonlinear coefficient $d_{31}$ with photobleaching time with $488 \mathrm{~nm}$ radiation was deduced. All the measurements were made in air where photo-oxidation contributes significantly to the photodegradation.

The results for $d_{31}$ for DANS are shown in Fig. 9. Clearly, photobleaching leads to a reduction in the second order nonlinearity. Also it is evident that there were two contributing processes involved, one of which bleaches out much faster than the second one. The variation of $B$ with wavelength has shown that the photobleaching figure of merit for the higher energy charge-transfer state dominates at this wavelength and we hypothesize that this is also the case for the nonlinear activity. What is surprising is that the two charge-transfer states seem to contribute equally to the second-order nonlinearity and these results merit further investigation. This is in contrast to other work on the reduction in the second-order nonlinearity in Disperse Red $1 .{ }^{30}$ In that case, there was a monotonic reduction in $d_{33}$ with illumination time, in keeping with the photobleaching measurements which indicated that only one dominant charge-transfer state was involved.

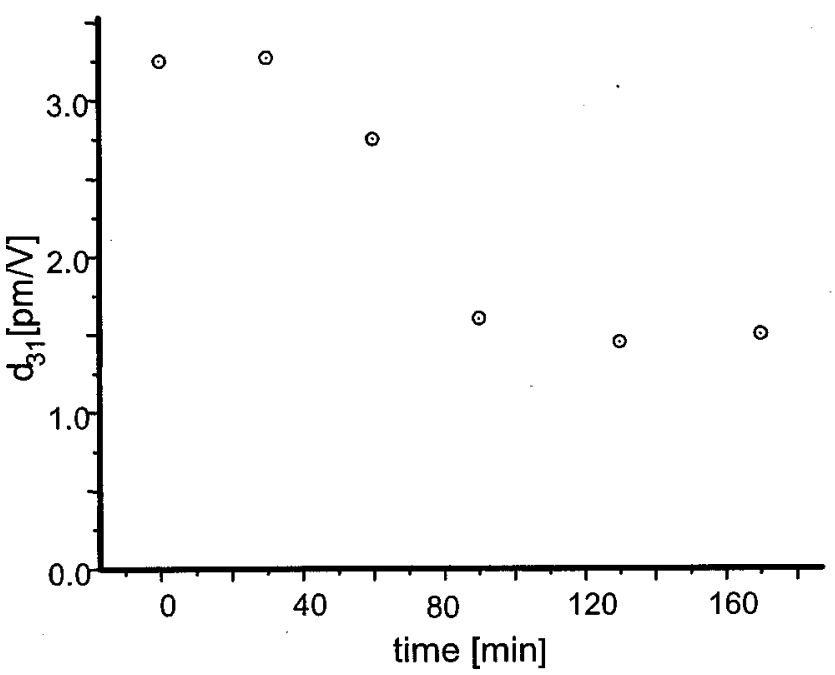

FIG. 9. Plot of $d_{31}$ vs time for DANS measured with $1579 \mathrm{~nm}$ radiation.

\section{CONCLUSIONS}

This study, albeit with a limited number of chromophores, has shown that this family of alkene and alkyne conjugated chromphores exhibits photostability inferior to the azobenzene family. The quantum efficiency for photodegradation is typically a factor 50 greater than for a typical azobenzene (and this would scale in lifetime of associated devices used is similar operating conditions). Thus, in general, the $-\mathrm{C}=\mathrm{C}-$ double bond appears to be, under our operating conditions, much more susceptible to undergo photoinduced transformations than the $-\mathrm{N}=\mathrm{N}-$ double bond. Nevertheless, if the absorption spectrum is sufficiently spectrally narrow, then the figure-of-merit for the best chromophores approach that of typical azobenzenes. These results suggest that future materials investigated for optical applications in integrated optics should employ measures needed to stabilize or protect the $\mathrm{C}=\mathrm{C}$ functionality. This outcome may be accomplished by a number of means. First, molecular structure modification will certainly still lead to intrinsic structure stability enhancement. Second, the important role of oxygen in the photodegradation must be mitigated. This may require the inclusion of stabilizers such as catalytic or even stoichiometric scavengers for triplet and singlet oxygen. Another obvious important component will be the elimination of oxygen by appropriate processing and packaging methods.

\section{ACKNOWLEDGMENTS}

The authors would like to acknowledge the financial support at CREOL and Arizona of NSF under a GOALI program, and at CREOL and IOTA by a CNRS/NSF French/US bilateral grant and at the California Institute of Technology from the Air Force Office of Scientific Research.

\footnotetext{
${ }^{1}$ Many articles in L. A. Hornak, Polymers for Lightwave and Integrated Optics: Technology and Applications (Marcel Dekker, New York, 1992); K. D. Singer and J. H. Andrews, in Molecular Nonlinear Optics, edited by Z. Zyss (Academic, San Diego, 1994), pp. 245-298; L. Dalton, Poly. Phot. Appl. I, 158, 1 (2002).
} 
${ }^{2}$ W. H. G. Horsthuis, G. R. Möhlmann, and H. W. Mertens, Digest of the Conference on Nonlinear Guided-Wave Phenomena (OSA, Cambridge, 1991), MD1, pp. 70-73.

${ }^{3}$ M. B. J. Diemeer et al., Electron. Lett. 26, 379 (1990).

${ }^{4}$ C. C. Teng, Appl. Phys. Lett. 60, 1538 (1992).

${ }^{5}$ For example, T. E. Van Eck et al., Appl. Phys. Lett. 58, 1588 (1991).

${ }^{6}$ R. A. Norwood, D. R. Holcomb, and F. F. So, Nonlinear Opt. 6, 193 (1993).

${ }^{7}$ A. Zweig and W. J. Henderson, J. Polym. Sci. Polym. Chem. Ed. 13, 717 (1975).

${ }^{8}$ M. A. Mortazavi et al., Polym. Prepr. (Am. Chem. Div. Polym. Chem.) 35, 198 (1994).

${ }^{9}$ A. Mortazavi, H. N. Yoon, and C. C. Teng, J. Appl. Phys. 74, 4871 (1993).

${ }^{10}$ T. Zyung, W. Y. Hwang, and J. J. Kim, Appl. Phys. Lett. 64, 3527 (1994).

${ }^{11}$ Q. Zhang, M. Canva, and G. I. Stegeman, Appl. Phys. Lett. 73, 912 (1998).

${ }^{12}$ A. Galvan-Gonzalez, M. Canva, and G. I. Stegeman, Appl. Phys. Lett. 75, 3306 (1999).

${ }^{13}$ M. Cha et al., Appl. Phys. Lett. 65, 2648 (1994).

${ }^{14}$ D. H. Waldeck, Chem. Rev. (Washington, D.C.) 91, 415 (1991).

${ }^{15}$ O. V. Butrimovich et al., Opt. Spectrosc. 69, 343 (1990); translated from Opt. Spektrosk. 69, 574 (1990).

${ }^{16}$ D. Tomic and A. Mickelson, Appl. Opt. 38, 3893 (1999).

${ }^{17}$ A. Dubois, M. Canva, A. Brun, F. Chaput, and J.-P. Boilot, Appl. Opt. 35, 3193 (1996).
${ }^{18}$ A. Galvan-Gonzalez et al., Opt. Lett. 25, 332 (2000).

${ }^{19}$ A. Galvan-Gonzalez et al., Opt. Lett. 24, 1741 (1999).

${ }^{20}$ J. J. Kim, T. Zyung, and W. Y. Hwang, Appl. Phys. Lett. 64, 3488 (1994).

${ }^{21}$ R. S. Moshrefzadeh et al., Appl. Phys. Lett. 62, 16 (1993).

${ }^{22}$ J. Ma et al., Appl. Opt. 34, 5352 (1995).

${ }^{23}$ A. Chen et al., Presented at the Organic Thin Films for Photonics Applications Technical Digest Vol. 14, Washington, DC, 1997 (unpublished).

${ }^{24}$ A. Galvan-Gonzalez, M. Canva, G. I. Stegeman, L. Sukhomlinova, R. J. Twieg, K. P. Chan, T. C. Kowalczyk, and H. S. Lackritz, J. Opt. Soc. Am. B 17, 1992 (2000).

${ }^{25}$ D. Beljonne, J. L. Bredas, M. Cha, W. E. Torruellas, G. I. Stegeman, W. H. G. Horsthuis, and G. R. Mohlmann, J. Chem. Phys. 103, 7834 (1995).

${ }^{26}$ A. Galvan-Gonzalez, K. D. Belfied, G. I. Stegeman, M. Canva, K. P. Chan, K. Park, L. Sukhomlinova, and R. J. Twieg, Appl. Phys. Lett. 77, 2083 (2000).

${ }^{27}$ K. D. Belfield, C. Chinna, and K. J. Schafer, Tetrahedron Lett. 38, 6131 (1997).

${ }^{28}$ A. Otomo, M. Jäger, G. I. Stegeman, M. C. Flipse, and M. Diemeer, Appl. Phys. Lett. 69, 1991 (1996).

${ }^{29}$ For example, J. Jerphagnon and S. K. Kurtz, J. Appl. Phys. 41, 1667 (1970).

${ }^{30}$ M. Nakanishi, O. Sugihara, N. Okamoto, and K. Hirota, Appl. Opt. 37, 1068 (1998). 